\title{
Improving the Accuracy of Iris Recognition System using Neural Network and Particle Swarm Optimization
}

\author{
Nuzhat Faiz Shaikh, \\ Department of Computer Engineering, \\ M.E.S. College of Engineering, Pune, India
}

\author{
D. D. Doye, Ph.D \\ Department of E\&TC, \\ SGGS IET, Nanded, India
}

\begin{abstract}
Iris recognition is the process of recognizing a person by analyzing the apparent pattern of his or her iris. Many techniques have been developed for iris recognition so far. Here, we propose a new iris recognition system with the help of local histogram and then optimized with FFBNN-PSO. In the proposed system, first the input eye images are preprocessed using adaptive median filter to remove the salt and pepper noise. Then, the features, which are extracted from the preprocessed image are given to FFBNN for training. In order to get accurate results, the FFBNN parameters are optimized using PSO.
\end{abstract}

\section{General Terms}

Soft Computing, Biometric.

\section{Keywords}

Feed Forward Back propagation Neural Network (FFBNN), Adaptive Median Filter, Feature Extraction, Iris Recognition, Particle Swarm Optimization (PSO).

\section{INTRODUCTION}

The texture of the human iris does not change. From one year of age until death, the patterns of the iris are comparatively constant over a person's lifetime. Because of this individuality and stability, iris recognition is a reliable human identification technique [1].

The iris is a membrane in the eye, responsible for controlling the amount of light reaching the retina [2]. The iris consists of pigmented fibro vascular tissue known as a stoma. It is the frontal portion of the eye and the only one seen on superficial inspection. Iris recognition is one of the most reliable biometric recognition approach.Ithas become a very active topic in research and practical application [3]. Not to be confused with another, less prevalent, ocular-based technology, i.e. retina scanning, iris recognition uses camera technology, with subtle infrared illumination reducing specular reflection from the convex cornea, to create images of the detail-rich, intricate structures of the iris [4] [5]. This turf is sometimes called iris texture. The iris texture provides many minute characteristics such as freckles, coronas, stripes, furrows, crypts, etc. These visible physiognomies are unique for each subject. Such unique features in the anatomical structure of the iris facilitates the difference among individuals [6]

The authors in [7] propose a fusion method for recognition of iris. After the iris has been identified using cumulative sum and euler number, the scores are fused to get better performance

Neural networks model biological neural networks in the brain and have proven their efficiency in a number of applications such as organization and categorization, prediction, pattern recognition and control [8]. Many types of neural networks exist including feed forward neural networks, radial basis function (RBF) networks, Kohonen selforganizing networks, recurrent networks, stochastic neural networks, modular neural networks, dynamic neural networks, cascading neural networks, and fuzzy neuro networks [9] [10].

The objective of this research is to develop an optimized NN model that can be used for by accurate iris recognition systems and provide better results as compared to already existing systems. [11][12][13].

\section{THE PROPOSED IRIS RECOGNITION SYSTEM}

In the proposed methodology, the given input image is preprocessed using adaptive median filter for removing salt and pepper noise. Then the features of the iris and pupil are extracted from the preprocessed imageThese extracted features are given to Feed Forward Back Propagation Neural Network (FFBNN) for training purpose. While training the parameters of the FFBNN are optimized using PSO. Then the output obtained from the feature extraction process is given to a well-trained FFBNN-PSO to validate whether the given input iris image is recognized or not. The architecture diagram of the proposed Iris Recognition System is shown in Fig. 1.

The Proposed iris recognition system consists of 3 stages:

1. Preprocessing

2. Feature Extraction

3. Recognition by FFBNN-PSO 


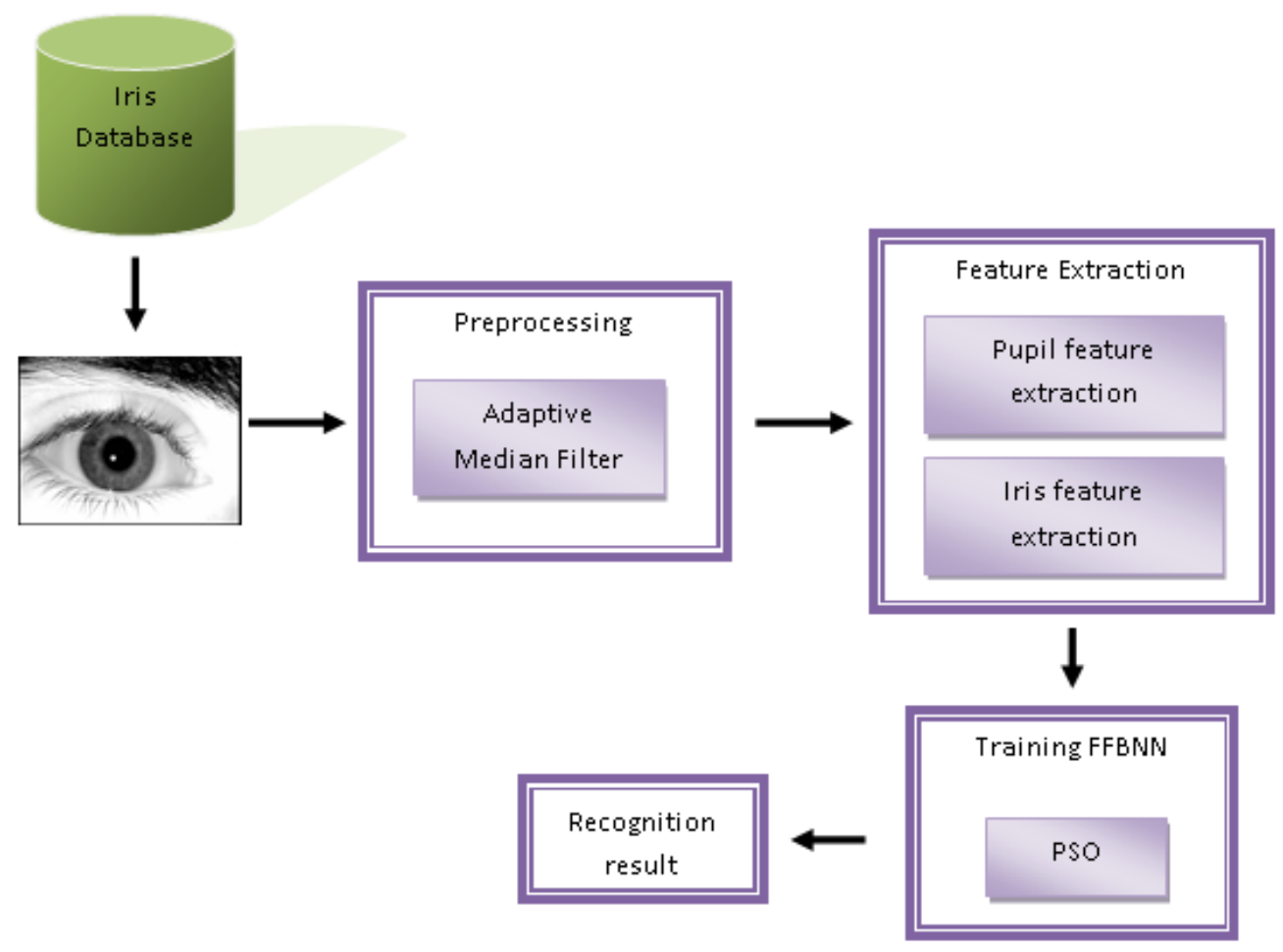

Figure 1: Architecture of proposed iris recognition system

\subsection{Preprocessing}

The input eye image is first changed into grey level format. Then the grey level eye image is preprocessed using Adaptive median filter to remove salt and pepper noise. This adaptive median filter works based on the local statistical characters. It detects the impulse by calculating the difference between the standard deviation of the pixels within the filter window and the concerned current pixel.

Process of Adaptive median filter

The working procedure of Adaptive median filtering is described below,

Initialize the window size $\boldsymbol{W}=3$.

Calculate maximum $\left(s \boldsymbol{d}_{i, j}^{\min , w}\right)$, minimum $\left(s d_{i, j}^{\max , w}\right)$ and median $\left(s d_{i, j}^{m e d, w}\right)$ of the pixel values in $s d_{i, j}^{w}$.

If $s d_{i, j}^{\min , w}<s d_{i, j}^{\operatorname{med}, w}<s d_{i, j}^{\max , w}$, then go to step 5 .

Otherwise increment the window size $\boldsymbol{w}$ by 2 .

If $w \leq w_{\text {max }}$ go to 2 . Otherwise replace $y_{i, j}$ by $s d_{i, j}^{m e d, w_{\max }}$

If $s d_{i, j}^{\min , w}<y_{i, j}<s d_{i, j}^{\max , w}$, then $y_{i, j}$ is not a noise

candidate otherwise replace $y_{i, j} s d_{i, j}^{m e d, w}$.
In the above adaptive median filter algorithm, the noise candidates only replaced by the median $s d_{i, j}^{m e d, w}$, while remaining are unaltered. Using the above adaptive median filter algorithm the salt and pepper noise is removed from the given input eye image and the preprocessed eye image is denoted as $x^{\prime}$. This preprocessed eye image $\left(x^{\prime}\right)$ is then subjected to feature extraction process.

\subsection{Feature Extraction}

Generally an image consists of pupil, eye lashes, sclera, eyelid, iris and skin. These parts are differing from each other based on their characteristics and grey level too. Here the features are extracted from two parts namely,

1. Pupil Feature Extraction

2. Iris Feature Extraction

\subsubsection{Pupil Feature Extraction}

Before extracting the features of the pupil, the pupil must be segmented from the eye image. In order to localize the pupil from the preprocessed eye image $\left(x^{\prime}\right)$, the following process is carried out.

Step1. Create a circular window with radius $R$. The value of $R$ is calculated as

$$
R=0.30 \times m
$$

where $\boldsymbol{m}_{\text {is }}$ the number of rows of the given input image. The window is slided from the left most to the right most column of the image.

Step2. The circular window is placed on the image coordinates $\left(c_{r}, c_{c}\right)$. 
where $c_{r}$ - the central row of the image,

$c_{c}=R+\frac{R}{2} \times i$

If $c_{c}+R \leq n$,

In equ.(3),

$n$ - Number of columns of an input image.

Go to Step 3 else go to Step 11.

Step3. Calculate histogram of the masked area.

Step4. Compute the minimum and maximum grey levels of the masked area and threshold $\tau$

Step5. Find the coordinates $\left(x_{u}, y_{u}\right)$ corresponding to the maximum frequency in the lower grey level range $\left(l_{l}\right)$ which ranges from $\min$ grey to $\tau$. Also find $\left(x_{l}, y_{l}\right)$ corresponding to the upper grey level range $\left(u_{l}\right)$ which ranges from $\operatorname{ceil}(\tau)+1_{\text {to }} \max _{\text {grey }}$. Find the standard deviation of the coordinates:

Step6. Calculate the difference between the standard deviations:

$d_{u}=\left|s_{x_{u}}-s_{y_{u}}\right|, \quad d_{l}=\left|s_{x_{l}}-s_{y_{l}}\right|$.

Step7. If $d_{u}<d_{l}$

Set range $=u_{l \text { and }} P_{g}=m f_{u}$

Else range $=l_{\text {and }} P_{g}=m f_{l}$

Where,

$m f_{u}$ - grey level in the upper level which corresponds to the maximum frequencies

$m f_{l}$ - grey level in the lower level which corresponds to the maximum frequencies

$P_{g} \quad$ - grey level of the pupil region

Go to Step 4 until $P_{g}$ stops changing.

Step8. All the grey levels in the range from $P_{g}-\varepsilon_{\text {to }}$ $P_{g}+\varepsilon$ are declared as the grey levels of the pupil region to be oin the safer side. Based on experience $\mathcal{E}=5$.

Step9. Find the standard deviation ${ }^{s} p i$ for the pupil region's coordinates (white pixels).

Step11. Increment $i$ by 1 and continue Step 2 .
After localizing the pupil, the values of pixels which are lying

in the circle is stored in a variable $\boldsymbol{v} \boldsymbol{1}_{\text {for extracting the }}$ features from the pupil. Mean and Skewness of the pupil part is calculated which are given as the input to FFBNN.

The mean $(\mu \nu 1)$ can be calculated for pupil boundary using the formula:

$\mu v 1=\frac{1}{m 1} \sum_{i=0}^{m 1} v 1_{i}$

Where,

$m 1$ - Total number of values stored in $v 1$

$v 1_{i_{-}}$ith term value of $v 1$

The skewness value of pupil boundary $(s 1)$ is calculated as follows:

$s 1=\frac{1}{m 1} \sum_{i=o}^{m 1}\left(\frac{v 1_{i}-\mu v 1}{\sigma 1}\right)^{3}$

Where,

$\sigma 1_{\text {- standard deviation of }} \boldsymbol{v 1}$. It is given as

$\sqrt{\left(\frac{1}{m 1} \sum_{i=o}^{m 1}\left(v 1_{i}-\mu v 1\right)^{2}\right)}$

The image obtained after pupil extraction is then subjected to iris feature extraction.

\subsubsection{Iris Feature Extraction}

In order to extracting the features of the iris image, first the iris must be segmented from the pupil. Generally, the center of the iris lies within the pupil region. But the pupil's center and iris center are not coincident. So a circular region is selected whose radius is 3 times the pupil's radius which reduces the search space to locate the outer boundary of the iris. The other portions are neglected. Finding the edges of the iris from the central row is easier than from the central column. Due to the presence of eye lashes and eyelids in the partially opened eye, locating the outer boundary of the iris from the central column is not possible. The grey level of the central row is splitted into 3 regions. They are black, Iris and Sclera regions.

It is not possible to locate the correct boundary of the iris due to the presence of the eyelashes and eyelids by selecting the central row as the reference line. So reference line is selected from the below of the pupil's central row as candidate row to reduce the effect of eyelashes and eyelids. First the first order derivative of the candidate row is calculated which is used to detect the boundary of the iris. Then the left and right boundary points of the iris are calculated.

The pixels which are lying in the iris circle is stored in a variable $v 2$. Then the mean $(\mu v 2)$ and Skewness $(s 2)$ value is computed and it is given as the input to FFBNN.

After extracting the iris features, these iris and pupil features are given to FFBNN. 


\section{RECOGNITION BY FFBNN-PSO}

\subsection{Training the Neural Network}

For iris recognition, Feed Forward Back Propagation Neural Network (FFBNN) is trained using the mean and skewnessvalues extracted from the pupil and iris boundaries of the each and every image in the database. The extracted features are given to neural network for training and recognition purpose. The neural network is well trained using these extracted features. For training purpose, the image obtained after localization process are taken out and the features are extracted. The neural network consists of 4 input units, $\mathrm{h}$ hidden units and one output unit. The structure of the FFBNN is given as below:

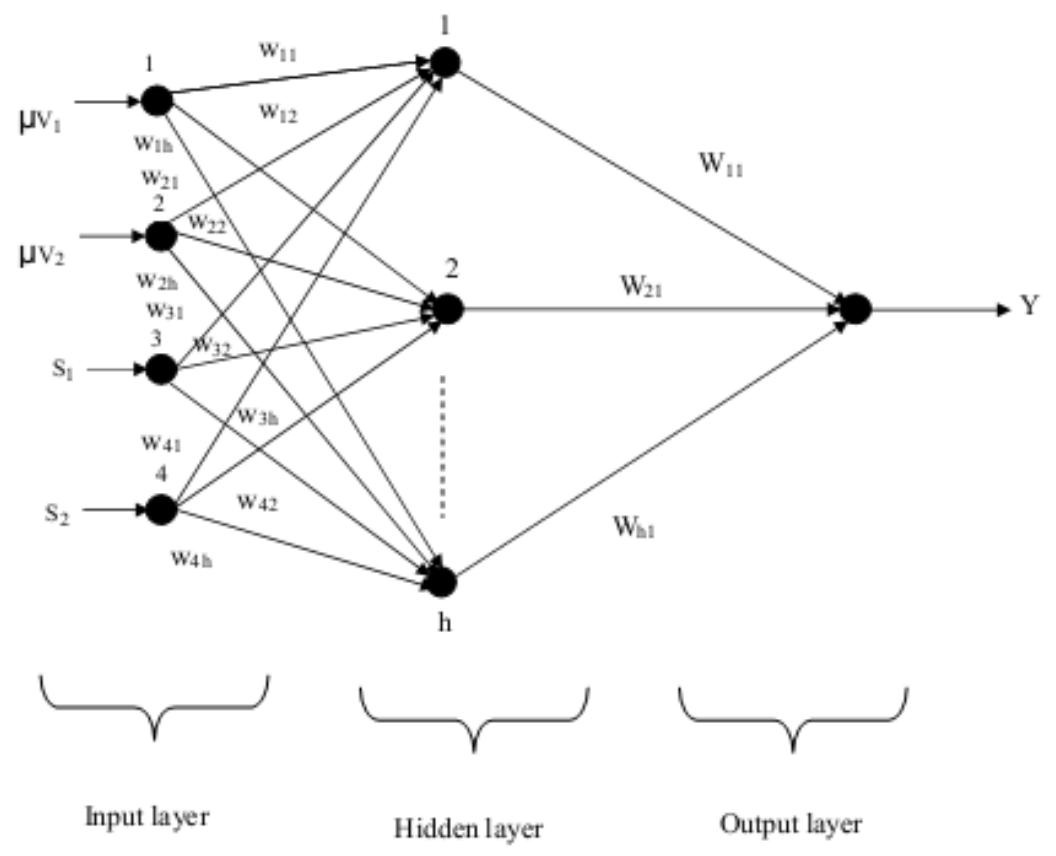

Figure 2.The proposed FFBNN with four input neurons and one output neuron

Assign weights randomly to all the neurons except input neurons.

The bias function and activation function for the neural network is described below.

$$
\begin{aligned}
& X(t)=\beta+\sum_{n=0}^{h-1}\left(\begin{array}{l}
w_{t n} \mu v 1_{t n}+w_{t n} \mu \nu 2_{t n}+w_{t n} s 1_{t n} \\
+w_{t n} s 2_{t n}
\end{array}\right) \\
& X(a)=\frac{1}{1+e^{-X(t)}}
\end{aligned}
$$

In bias function $\mu v 1_{t n}, s 1_{t n}, \mu v 2_{t n}$ and $s 2_{t n}$ are the extracted features of pupil and iris boundaries respectively. The activation function for the output layer is given in Eq. (8).

Find the learning error.

$E=\frac{1}{h} \sum_{n=0}^{h-1} d_{n}-a_{n}$

$E_{\text {is }}$ the FFBNN network output, $d_{n}$ and $a_{n}$ are the desired and actual outputs and $h$ is the total number of neurons in the hidden layer .

\subsection{Error Minimization:}

Weights are allocated to the hidden layer and output layer neurons by randomly chosen weights. The input layer neurons have a constant weight

Determine the bias function and the activation function.
Calculate BP error for each node and update the weights as follows:

$$
w_{(t n)}=w_{(t n)}+\Delta w_{(t n)}
$$

$\Delta w_{(t n)}$ is obtained as

$$
\Delta w_{(t n)}=\delta . X\left(t_{n}\right) . \text { Bpe }
$$

Where $\delta$ is the learning rate, which normally ranges from 0.2 to 0.5 , and $B p e$ is the BP error.

Then repeat the steps (2) and (3) until the BP error gets minimized. The process is repeated until it satisfies $B P e<0.1$ .If the error gets minimized to a minimum value the FFBNN is well trained for performing the testing phase.

Then the result of the neural network $(Y)$ is compared with the threshold value $(\tau 1)$. If it satisfies the threshold value it is recognized.

result $=\left\{\begin{array}{l}\text { recognized }, Y \geq \tau 1, \\ \text { not recognized }, Y<\tau 1\end{array}\right.$

\subsection{Particle swarm optimization}

Particle swarm optimization (PSO) is population based search algorithm. It is created to pretend the behavior of birds in search for food on a cornfield or fish school. The method can 
efficiently find optimal or near optimal solutions in large search spaces.

Each individual particle $i$ has a randomly initialized position $X_{i}=\left(x_{i}^{1}, x_{i}^{2}, \cdots, x_{i}^{D}\right)$ where $x_{i}^{d}$ being its position in the dth dimension, velocity, $V_{i}=\left(v_{i}^{1}, v_{i}^{2}, \cdots, v_{i}^{D}\right)$ where $v_{i}^{d}$ being the velocity in the $d$ th dimension, $p b_{i}=\left(p b_{i}^{1}, p b_{i}^{2}, \cdots, p b_{i}^{D}\right)$ where $p b_{i}^{d}$ being the best position in the dth dimension and $g b=\left(g b^{1}, g b^{2}, \cdots, g b^{D}\right)$ where $g b^{d}$ being the global best position in the dth dimension in the D-dimensional search space. Any particle can move in the direction of its personal best position to its best global position in the course of each generation. The moving process of a swarm particle in the search space is described as:

$V_{i}^{d}=V_{i}^{d}+c_{1} \cdot r_{1} \cdot\left(p b_{i}^{d}-x_{i}^{d}\right)+c_{2} \cdot r_{2} \cdot\left(g b^{d}-x_{i}^{d}\right)$

$x_{i}^{d}=x_{i}^{d}+\delta V_{i}^{d}$

In equation (13),

$c_{1}, c_{2}$ - constants with the value of 2.0

$r_{1}, r_{2}$ - independent random numbers generated in the range [0.1]

$V_{i}^{d}$ - velocity of ith particle

$x_{i}^{d}$ - current position of the particle $i$

$p b_{i}^{d}$ - best fitness value of the particle at the current iteration

$g b^{d}$ - best fitness value in the swarm.

D. FFBNN parameter optimization by PSO

Swarm initialization: For a population size $\mathrm{m}$, generate the particles randomly.

Define the fitness function: The fitness function choosen which should be used for the constraints according to the current population. Here eqn. (8) is used for fitness function calculation.

$$
g b_{\text {and }} p b_{\text {Initialization: }}
$$

Initially the fitness value calculated for each particle is set as the Pbest value of each particle. Among the Pbest values, the best one is selected as the $g \boldsymbol{b}$ value.

- $\quad$ Use c1 and c2 to be equal to 2 .

- Use equation 13 to compute the velocity

Swarm Updation: Calculate the fitness function again and update the $p b$ and $g b$ values. If the new value is better than the previous one, replace the old by the current one. And also select the best $p b$ as the $g \boldsymbol{b}$.

Criterion to stop: Continue until the solution is good enough or maximum iteration is reached.

This optimization of FFBNN parameters using PSO gives higher recognition result and effective performance. In the testing process, first the given input testing image is preprocessed using the adaptive median filter. Then features are extracted by localizing the pupil and iris of the preprocessed eye image. Then the extracted features are given to well trained FFBNN-PSO to evaluate whether the given image is recognized or not.

\section{EXPERIMENTAL RESULTS}

The proposed iris recognition system with FFBNN-PSO is implemented with the help of MATLAB (version 7.12) with machine configuration as follows

Processor: Intel core i7

OS: Windows 7

CPU speed: $3.20 \mathrm{GHz}$

RAM: 4GB

The performance of the proposed iris recognition system is evaluated with the help of 50 number of iris images which are taken from the UBIRIS database [14].

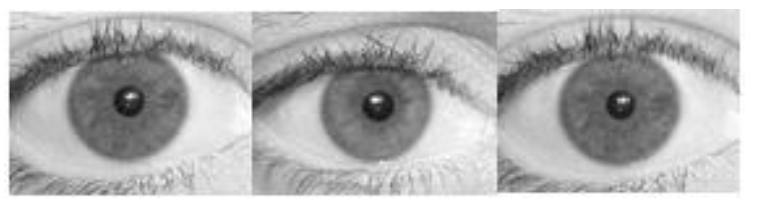

Figure 3: Sample iris images

To remove the noise from the iris images, the images are given to the AMF. These preprocessed images are then subjected to the feature extraction process. While feature extraction, the pupil and iris features are extracted from the preprocessed images and that images are given to neural network for training process. While training, the FFBNN parameters are optimized using AAPSO.

\section{Performance Analysis:}

The database images are divided into five datasets, each consisting of 10 images. The performance of our proposed iris recognition system using PSO, is evaluated and compared with existing system [13] and FFBNN.

Table 1: Proposed, existing iris recognition systems [13], FFBNN-PSO and FFBNN iris recognition methods performance results in terms of (i) Accuracy (ii) Sensitivity and (iii) Specificity

\begin{tabular}{|l|l|l|l|}
\hline Measures & $\begin{array}{l}\text { Existing } \\
\text { System [13] }\end{array}$ & $\begin{array}{l}\text { FFBNN- } \\
\text { PSO }\end{array}$ & FFBNN \\
\hline Accuracy & 79.9 & 80 & 72 \\
\hline Sensitivity & 80 & 88 & 68 \\
\hline Specificity & 100 & 76 & 76 \\
\hline
\end{tabular}




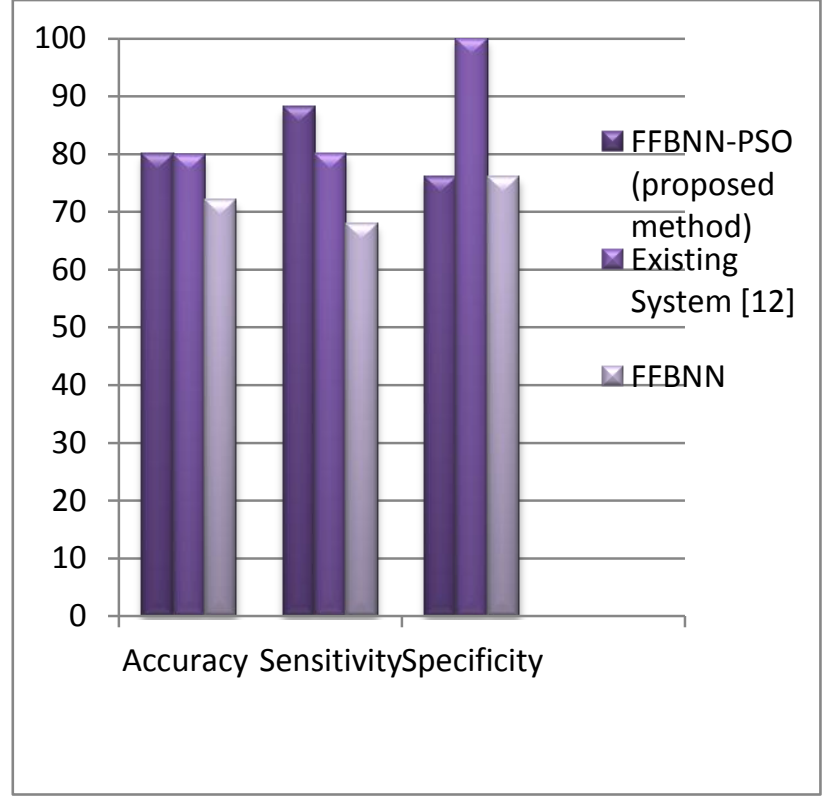

Figure 4: Proposed(FFBNN-PSO), existing iris recognition systems [13], and FFBNN iris recognition methods performance results in terms of (i) Accuracy (ii) Sensitivity and (iii) Specificity

\section{CONCLUSION}

In this paper, we have proposed an iris recognition system based on FFBNN and PSO. The proposed system was implemented and a huge set of test iris images were utilized to analyze the results of the proposed iris recognition system. The performance analysis as shown in Table 1 and Figure 4, proved that the proposed system which uses PSO to optimize the FFBNN parameters, offers better accuracy as compared to the system that uses traditional FFBNN. When compared with existing system[13], if not better, the results are comparable. We propose to improve the performance parameters by further optimization and modification in the constants $\mathrm{c} 1$ and $\mathrm{c} 2$ of PSO as future work.

\section{REFERENCES}

[1] Li Ma, Tieniu Tan, Yunhong Wang and Dexin Zhang, "Efficient Iris Recognition by Characterizing Key Local Variations", IEEE Transactions on Image Processing, Vol. 13, No. 6, pp. 739-750, June 2004.

[2] Jiali Cui, Yunhong Wang, Tieniu Tan, Li Ma and Zhenan Sun, "A fast and robust iris localization method based on texture segmentation", In proceedings of conference on Biometric Technology for Human Identification, China, Vol. 5401, pp. 1-8, 2004.

[3] Leila FallahAraghi, HamedShahhosseini and FarbodSetoudeh, "IRIS Recognition Using Neural
Network", In proceedings of the International Multi Conference of Engineers and Computer Scientists, Vol. 1, Hong Kong, pp. 1-3, 2010.

[4] Dolly Choudhary, ShamikTiwari, Ajay Kumar Singh, "A Survey: Feature Extraction Methods for Iris Recognition", International Journal of Electronics Communication and Computer Technology, Vol. 2, No. 6, pp. 275-279, 2012.

[5] Hind Rostom Mohammed, "Gout Images Detection and Recognition by Neural Network", Journal of university of anbar for pure science, Vol. 5, No. 2, pp. 1-5, 2011.

[6] RahibH.Abiyev and KorayAltunkaya, "Personal Iris Recognition Using Neural Network", International Journal of Security and its Applications, Vol. 2, No. 2, pp. 41-50, 2008.

[7] N.F. Shaikh, Dr. D. D. Doye, "Combining the Goodness of Euler Number and Cumulative Sum to Achieve Higher Accuracy for Iris Recognition Systems", IJETCAS, Issue 5 Volume 2, pp. 183-188, June-August, 2013

[8] Chowhan and Shinde, "Iris Recognition Using Fuzzy Min-Max Neural Network", International Journal of Computer and Electrical Engineering, Vol. 3, No. 5, pp. 743-749, 2011.

[9] Gasser Auda and Mohamed Kamel, "CMNN: Cooperative Modular Neural Networks for pattern recognition", Pattern Recognition Letters, Vol. 18, No. 11, pp. 1391-1398, 1997.

[10] Omaima N. Ahmad AL-Allaf, AbdelfatahArefTamimi and Shahlla A. AbdAlKader "Artificial Neural Networks for Iris Recognition System: Comparisons between Different Models, Architectures and Algorithms", International Journal of Information and Communication Technology Research, Vol. 2, No. 10, pp. 744-752, 2012.

[11] Sun-Yuan Kung and Jenq-Neng Hwang, "Neural networks for intelligent multimedia processing", In proceedings of the IEEE, Vol. 86, No. 6, pp. 1244-1272, 1998.

[12] Ahmad M. Sarhan, "Iris Recognition Using Discrete Cosine Transform and Artificial Neural Networks", Journal of Computer Science, Vol. 5, No. 5, pp. 369-373, 2009.

[13] NareshBabu and Vaidehi, "Fuzzy Based IRIS Recognition System (FIRS) For Person Identification", In Proceedings of the IEEE-International Conference on Recent Trends in Information Technology, Chennai, Tamil Nadu, pp. 1005-1011, 2011

[14] http://iris.di.ubi.pt/ UBIRIS iris database 